\title{
La ciencia española a través de la Web of Science (1996-2007): las disciplinas
}

\author{
Por Emilio Delgado-López-Cózar, Evaristo Jiménez-Contreras y Rafael Ruiz-Pérez
}

\begin{abstract}
Resumen: A partir de la información suministrada de manera gratuita por Thomson Reuters en ScienceWatch.com se presentan datos sobre la producción y citación de las distintas disciplinas científicas que conforman la ciencia española. Se determina el peso relativo que tiene cada una de ellas tanto en el ámbito nacional como en el internacional a lo largo de los últimos 10 años

Palabras clave: Bibliometría, Análisis de citas, Publicación científica, Evaluación del rendimiento investigador, Thomson Reuters, ScienceWatch.com, National science indicators, Disciplinas, España.
\end{abstract}

\section{Title: The Spanish science as seen through the Web of Science (1996-2007): the disciplines}

Abstract: From the information provided for free by Thomson Reuters in ScienceWatch.com, production and citation data of the scientific disciplines are analyzed for Spain, determining the relative weight of each one both nationally and internationally throughout the past 10 years.

Keywords: Bibliometrics, Citation analysis, Scientific publication, Scientfic perfomance, Thomson Reuters, ScienceWatch.com, National science indicators, Research fields, Spain.

Delgado-López-Cózar, Emilio; Jiménez-Contreras, Evaristo; Ruiz-Pérez, Rafael. "La ciencia española a través del Web of Science (1996-2007): las disciplinas". El profesional de la información, 2009, julio-agosto, v. 18, n. 4, pp. 437443.

DOI: 10.3145/epi.2009.jul.13

\section{Introducción}

A LA LUZ DE LAS CIFRAS que presentamos en el artículo anterior (Delgado et al. 2009) podemos retrucar el eslogan que popularizaran nuestros dos últimos presidentes del gobierno: la ciencia en España no va bien, va mejor. Y si no fuera por la situación de crisis en la que está sumido el país, las cifras apuntarían a que lo mejor estaría por venir.

Ahora bien, hecho este primer diagnóstico cabe hacerse una pregunta más: ¿ha ido igual de bien en todos los campos de conocimiento? Pues bien, en este artículo intentaremos responder a esta pregunta $\mathrm{e}$ indagar de paso alguna cuestión relacionada como la apertura exterior y el grado de pujanza internacional de los sectores que conforman la actividad científica española.

\section{Fuente de datos}

Como ya se señaló en el primer artículo de esta serie (Ruiz et al., 2008) Thomson Reuters (TR) viene ofreciendo información bibliométrica de forma gratuita a través del portal ScienceWatch.com. Desde hace ya ocho años (2001) TR publica anualmente una radiografía de la producción e impacto de la ciencia de distintos países, entre ellos España, vista a través de la Web of Science (WoS) y organizada por disciplinas científicas. La información se extrae de los NSI (National Science Indicators), producto de pago no incluido dentro de la licencia del Web of Knowledge, donde figuran el número de trabajos publicados y las citas generadas cada año por más de 180 países.

Los datos se presentan en series temporales de cinco años. La ventana de datos se actualiza con la incorporación del último año (1996-2001, $1997-$ 2002, 1998-2003...). Con ello se dota de una gran estabilidad a los datos, evitando las habituales distorsiones a que se ven sometidas las series anuales, y permitiendo trazar la evolución de fondo de cada especialidad de forma bastante precisa.

Supuesto que la finalidad de estos informes es determinar qué campos científicos dentro de un país crecen o decrecen a lo largo del tiempo, la decisión metodológica clave es la clasificación empleada y, particularmente, el número de categorías elegidas para agrupar las especialidades científicas en que se estructura el conocimiento científico. En la actualidad, $T R$ utiliza 24 categorías, aunque para el período aquí estudiado el número era de 22. Conocer el contenido de cada una de ellas es vital para poder interpretar atinadamente los datos (cuadro 1). Una rápida ojeada al cuadro de clasificación nos muestra dos importantes lagunas que deben ser tenidas en cuenta:

1. No se ofrece información sobre Arte y Humanidades.

2. No se presenta información desagregada para las Ciencias Sociales que aparecen todas mezcladas indiscriminadamente en una sola categoría, a excepción de la Economía y la Psicología/Psiquiatría, que cuentan con la suya propia.

Estas limitaciones no son más que otra manifestación de un sesgo bien conocido de las bases de da- 


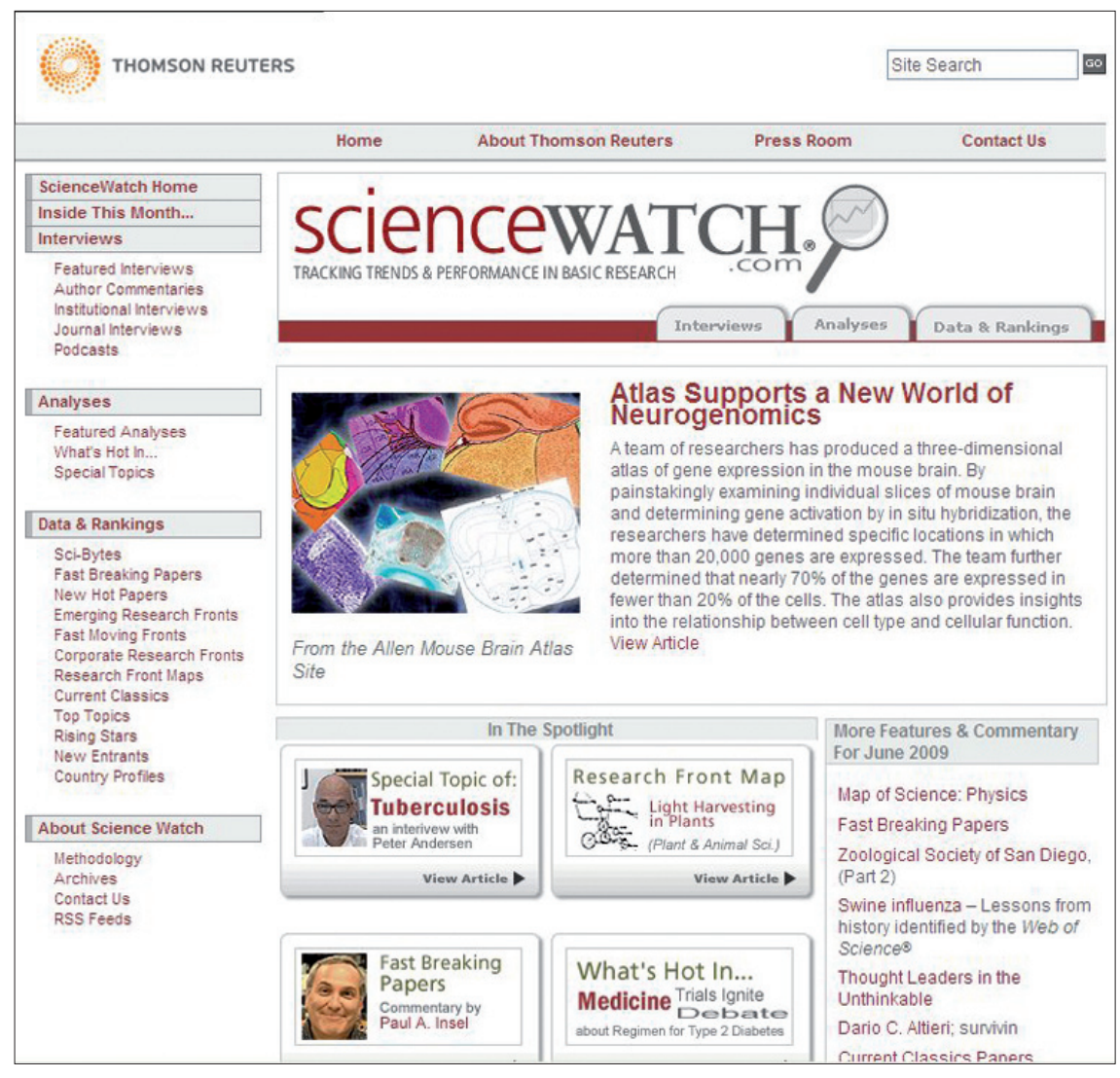

http://www.sciencewatch.com

tos de $T R$ : una clara inclinación por la ciencia básica, en general, y por disciplinas como Física, Química, Matemáticas, Biología, y Ciencias Médicas, en particular, que se encuentran ampliamente cubiertas en detrimento de las áreas aplicadas y tecnológicas, de las ciencias sociales y especialmente de las humanas, que están infra representadas.

Recuérdese que los revistas indizadas en las bases de datos WoS corresponden en un 69\% al área de Ciencia y Tecnología, en un $20 \%$ al de Ciencias sociales y en un $11 \%$ a las Humanidades. Conviene subrayar por tanto el moderado valor que posee este producto para reflejar la situación de las disciplinas de Ciencias sociales (excepto para la Psicología y la Economía), limitación que se acrecienta aún más en el caso de las humanísticas.

Otro aspecto metodológico importante en este tipo de trabajos es el relativo al sistema de asignación y de recuento empleado para la unidad de análisis. En la elaboración de pueden inflarse y otras deshincharse de forma más o menos artificial y se termina trasladando una imagen distorsionada de la realidad (Jiménez et al. 2005). Así por ejemplo, en la categoría, Information science and library science, el $55 \%$ de las revistas está sólo en esta categoría y en ninguna otra más, pero en Psychology es casi el 75\%; esto es, la categoría IS\&LS depende casi en un $50 \%$ de revistas que están en otras categorías, lo que abulta de una forma en cierta medida artificial sus resultados, mientras que en Psychology la dependencia de otras categorías es tan sólo del $25 \%$ y la distorsión por tanto es mucho menor.

\section{Resultados}

En los informes sobre situación de las disciplinas científicas de cada país publicados en ScienceWatch. com, TR emplea sólo dos medidas:

1. Para la producción: Porcentaje de artículos que en el campo científico de que se trate provienen del país analizado; es decir, el 100 representaría al mundo, aunque, de hecho, debido a la colaboración internacional, la suma de los porcentajes de todos los países arroja un total muy superior a 100. Una definición más estricta sería que el valor represente el tanto por ciento de trabajos en los que el país ha participado sin definir el nivel de dicha participación (el país puede ser responsable único del trabajo o haber colaborado con otros muchos sin que este extremo quede determinado).

2. Impacto: A partir del promedio de citas por trabajo en cada macro especialidad, se calcula el impacto relativo de cada categoría medido como desviación en porcentaje respecto a la media mundial en dicha categoría. En este caso un valor 0 no significa un impacto 0 sino que el área tiene un impacto igual a la media mundial, un valor positivo expresa la desviación porcentual con respecto a esa media y uno negativo lo contrario. 


\section{AGRI CULTURAL SCIENCES}

agricultural engineering, agronomy, tillage research, agroforestry, horticulture, crop protection and science, agrochemistry, phytochemistry, agricultural biochemistry, food chemistry, cereal chemistry, carbohydrate and lipid research, food science and nutrition, composition, additives and contaminants, microbiology and technology, engineering and processing, meat and dairy science, nutrition science, nutrition and metabolism, nutritional biochemistry

\section{BI OLOGY \& BI OCHEMI STRY}

structure and chemistry of biological molecules, molecular, cellular and clinical studies of the endocrine system, regulation of cell, organ and system functions by hormones, experimental research in general biology and biological systems, regulation of biological functions at the whole organism level, exploitation of living organisms or their components, industrial microbiology, pollution remediation, industria chemicals and enzymes, biosensors, bioelectronics, pesticide development, food, flavor and fragrance industry applications, waste treatment

\section{CHEMI STRY}

analytical chemistry, spectroscopy, instrumentation, inorganic and nuclear chemistry, organic chemistry, physical chemistry, polymer science, food chemistry, chemical methods and structures, natural and laboratory syntheses, isolation and analysis of clinically significant molecules, medicinal chemistry, chemical engineering

\section{NI CAL MEDI CI NE}

anaesthesia, cardiovascular medicine, dentistry, dermatology, general \& internal medicine, endocrinology, environmental medicine, gastroenterology, gynecology, hepatology, hematology, nephrology, nuclear medicine, obstetrics, oncology, ophthalmology, otolaryngology, pediatrics, pharmacology, radiology, toxicology, respiratory medicine, rheumatology, surgery, urology

\section{COMPUTER SCI ENCE}

computer software, software engineering and design, computer graphics, programming languages, theoretical computing, computing methodologies, broad computing topics, interdisciplinary computer applications, information systems and information technology, acquisition, processing, storage, management, and dissemination of information, communications via various devices and systems

\section{ENVI RONMENT/ ECOLOGY}

pure and applied ecology, ecological modeling and engineering, ecotoxicology, evolutionary ecology, environmental contamination and toxicology, environmental health, environmental monitoring and management, environmental technology, environmental geology, soil science and conservation, water resources research and engineering, climate change, biodiversity conservation. Natural history journals are also included here

\section{ECONOMI CS \& BUSINESS}

business, finance, management, organizational science, strategic planning and decision-making methods, industrial relations and labor ENGI NEERI NG

aerospace engineering, mechanical engineering, nuclear energy, electrical and electronics engineering, civil engineering, water resources and supply, transportation, and municipal engineering, effects of humans on the environment, controls to minimize environmental degradation, artificial intelligence, robotics and automatic control, engineering mathematics, mathematical modeling, optimization techniques, statistical methods in engineering systems, development, manufacture, and application of instruments

\section{GEOSCI ENCES}

geology, geochemistry, geophysics, geotechnics, economic geology, petrochemistry, mineralogy, meteorology and atmospheric sciences, hydrology, oceanography, petroleum geology, volcanology, seismology, climatology, paleontology, remote sensing, geodesy, geological, petroleum and mining engineering

\section{MMUNOLOGY}

clinical research in immunopathology, infectious diseases, autoimmunity and allergy, host-pathogen interactions in infectious disease, experimental therapeutic applications of immunomodulating agents

\section{MATERI ALS SCI ENCE}

ceramics, paper and wood products, polymers, textiles, composites, coatings \& films, biomaterials, metals and alloys, metallurgy, application of chemistry to materials design and testing, superconductors and semiconductors, ferroelectrics, dielectrics

MATHEMATI CS

applied mathematics, statistics and probability

MI CROBIOLOGY

biology \& biochemistry of microorganisms (bacterial, viral and parasitic), medical implications of the subsets of these organisms known to cause diseases, biotechnology applications of microorganisms for basic science or clinical use

\section{MOLECULAR BI OLOGY \& GENETI CS}

biochemistry, molecular biology, biophysics, pharmacology, receptor biology, signal transduction, regulation of gene expression, developmental genetics and biology, morphogenesis, cell-environment interactions, molecular genetics, mechanisms of mutagenesis, structure, function and regulation of genetic material, clinical genetics, patterns of inheritance, genetic causes, and screening and treatment of diseases

\section{MULTI DI SCI PLI NARY}

Includes journals of a broad or general character in the sciences and covers the spectrum of major scientific disciplines. It also includes journals devoted to a multidisciplinary approach to the study of particular regions, ecosystems or biological systems, and interdisciplinary journals designed to illuminate significant connections between fields.

\section{NEUROSCI ENCE \& BEHAVIOR}

cellular and molecular neuroscience, neuronal development, basic and clinical neurology, psychopharmacology, biobehavioral psychology, molecular psychology, neuronal function underlying higher cognitive processes

\section{PHARMACOLOGY}

pharmacology, pharmaceutics, cellular and molecular pharmacology, drug design and metabolism, mechanisms of drug action, drug delivery, natural products, xenobiotics, mechanisms of action for clinical therapeutics, toxicology, molecular and cellular effects of harmful substances, environmental toxicology, occupational exposure, clinical toxicology

\section{PHYSICS}

mathematical physics, particle and nuclear physics, physics of fluids and plasmas, quantum physics, theoretical physics, applied physics, condensed matter physics, physics of materials, optics and acoustics.

\section{PLANT \& ANI MAL SCI ENCE}

regional botany, mycology, bryology, plant physiology, forestry, weed science, plant pathology, economic botany, aquatic botany and toxicology, marine ecology, plant nutrition, photosynthesis research, experimental botany, cellular and molecular biology or physiology of plant cells and plant systems, animal behavior, animal production science, poultry science, wildlife research, lab animal science, zoology, primatology, mammalogy, herpetology, nematology, malacology, entomology and pest control, veterinary medicine, animal health, marine and freshwater biology, fisheries science, aquaculture

\section{PSYCHI ATRY/ PSYCHOLOGY}

psychology: applied, biological, clinical, developmental, educational, mathematical, organizational, personal, social, diagnosis and treatment

\section{SOCI AL SCI ENCES, GENERAL}

communication, environmental studies, library and information sciences, political science, public health and administration, rehabilitation, social work and social policy, sociology, anthropology, law, education

\section{SPACE SCI ENCES}

astronomy and astrophysics, celestial bodies, observation and interpretation of radiation from the component parts of the universe

Cuadro 1. Composición de las 22 categorías temáticas empleadas por Thomson Reuters 
Siguiendo este esquema presentamos dos tablas: la primera muestra la producción científica de cada disciplina indicando el porcentaje que representa respecto al total de la producción vaciada en la $W o S$ y la segunda indica el impacto relativo de cada disciplina comparada con la media mundial.

Tomado el porcentaje de producción alcanzada por un campo científico en el global de la base de datos WoS como indicador del volumen de la actividad científica en ese campo y, sobre todo, de su capacidad productiva y de su peso en la ciencia mundial, en la Tabla 1 se muestran los 22 campos científicos para España ordenados de acuerdo con su peso productivo. En todo caso convendría interpretar estos datos de producción también como un fiel reflejo de lo que son los hábitos de publicación de los distintos campos de conocimiento. De la lectura de esta tabla se desprenden los siguientes hechos:

\subsection{Más presencia de España}

El crecimiento sostenido de la producción española en el mundo (medio punto porcentual en ocho años) es un fenómeno que alcanza a todos los campos científicos. Todos han incrementado su peso en la producción científica mundial. Solo un campo científico (Farmacología) ha decrecido, aunque lo ha hecho sólo el 0,3\%. Los crecimientos más espectaculares han sido los de Economía $(110 \%)$, Ciencias Sociales (95\%), Informática $(88 \%)$ e Ingeniería $(74 \%)$. Dos son los factores que pueden explicar estos incrementos. El primero de ellos es la efectividad de la política de incentivos a la internacionalización de la ciencia española simbolizada por la
Cneai (Jiménez et al. 2003), que ha actuado especialmente en los campos menos expuestos al exterior (sociales y tecnológicos). El segundo, hay que atribuirlo a la progresiva incorporación de revistas españolas a los fondos de la WoS, espoleada por la política desarrollada en la $\mathrm{Fe}$ cyt (Pérez Andrés et al. 2006).

\subsection{España destaca en 8 de los 22 campos}

De las 22 categorías científicas sólo 8 figuran por encima de la tasa media de producción científica española en el mundo: Ciencias del espacio, Agricultura, Matemáticas, Microbiología, Química, Ciencia de las plantas y los animales, Ecología y Física. Esta cifra ha permanecido invariable a lo largo del período.

\subsection{Y de ellos especialmente en 4}

Cuatro son los campos científicos en los que podemos decir que

\begin{tabular}{|c|c|c|c|c|c|c|c|c|}
\hline Campo científico & $\begin{array}{c}1996- \\
2000 \\
\%\end{array}$ & $\begin{array}{c}1997- \\
2001 \\
\%\end{array}$ & $\begin{array}{c}1998- \\
2002 \\
\%\end{array}$ & $\begin{array}{c}1999- \\
2003 \\
\%\end{array}$ & $\begin{array}{c}2000- \\
2004 \\
\%\end{array}$ & $\begin{array}{c}2001- \\
2005 \\
\%\end{array}$ & $\begin{array}{c}2002- \\
2006 \\
\%\end{array}$ & $\begin{array}{c}2003- \\
2007 \\
\%\end{array}$ \\
\hline Space science & 5,24 & 5,51 & 5,67 & 5,79 & 5,85 & 6,10 & 6,14 & 6,43 \\
\hline Agricultural sciences & 4,71 & 4,86 & 5,11 & 5,30 & 5,52 & 5,73 & 6,11 & 6,34 \\
\hline Mathematics & 4,18 & 4,42 & 4,53 & 4,65 & 4,82 & 4,86 & 4,88 & 4,95 \\
\hline Microbiology & 4,14 & 4,26 & 4,40 & 4,41 & 4,49 & 4,58 & 4,63 & 4,64 \\
\hline Chemistry & 3,94 & 4,06 & 4,13 & 4,25 & 4,23 & 4,26 & 4,28 & 4,31 \\
\hline Plant $\&$ animal sciences & 3,59 & 3,70 & 3,81 & 3,88 & 3,91 & 4,04 & 4,17 & 4,25 \\
\hline Ecology/Environmental & 3,19 & 3,30 & 3,31 & 3,42 & 3,58 & 3,65 & 3,87 & 4,15 \\
\hline Economics \& Business & 1,76 & 2,00 & 2,29 & 2,64 & 2,92 & 3,17 & 3,50 & 3,71 \\
\hline Physics & 2,82 & 2,92 & 3,01 & 3,14 & 3,12 & 3,18 & 3,21 & 3,24 \\
\hline Biology \& Biochemistry & 2,74 & 2,81 & 2,88 & 2,87 & 2,98 & 3,03 & 3,09 & 3,18 \\
\hline Engineering & 1,75 & 1,94 & 2,11 & 2,32 & 2,48 & 2,65 & 2,87 & 3,05 \\
\hline Computer science & 1,61 & 1,83 & 2,05 & 2,26 & 2,45 & 2,72 & 2,80 & 3,03 \\
\hline Neuroscience & 2,45 & 2,57 & 2,69 & 2,73 & 2,80 & 2,88 & 2,93 & 3,00 \\
\hline Molecular biology & 2,45 & 2,56 & 2,60 & 2,66 & 2,75 & 2,76 & 2,80 & 2,94 \\
\hline Immunology & 2,63 & 2,56 & 2,61 & 2,57 & 2,68 & 2,71 & 2,80 & 2,91 \\
\hline Pharmacology & 2,91 & 2,96 & 2,82 & 2,86 & 2,91 & 2,95 & 2,95 & 2,90 \\
\hline Materials science & 2,54 & 2,64 & 2,82 & 2,90 & 2,90 & 2,89 & 2,97 & 2,89 \\
\hline Geosciences & 2,25 & 2,36 & 2,48 & 2,51 & 2,55 & 2,66 & 2,78 & 2,85 \\
\hline Clinical medicine & 2,41 & 2,47 & 2,54 & 2,54 & 2,54 & 2,60 & 2,65 & 2,69 \\
\hline Psychology/Psychiatry & 1,53 & 1,63 & 1,76 & 1,91 & 2,03 & 2,00 & 2,15 & 2,27 \\
\hline Social sciences & 0,61 & 0,70 & 0,75 & 0,84 & 0,89 & 1,06 & 1,11 & 1,19 \\
\hline \% total España & 2,75 & 2,86 & 2,95 & 3,02 & 3,08 & 3,18 & 3,25 & 3,32 \\
\hline
\end{tabular}

Tabla 1. Porcentaje de artículos publicados por España en la WoS distribuidos según campos científicos (1996-2007) 
España descuella a nivel mundial: Ciencias del Espacio (Astrofísica y Astronomía, básicamente), Agricultura, Matemáticas y Microbiología. Todas ellas alcanzan un porcentaje superior al 4,5\%, muy por encima del $3 \%$ de media en España para todo el período. Además, su situación es muy estable a lo largo del período, pues tanto en el primer (1996-2000) como en el último quinquenio del análisis (2003-2007) seguían siendo los principales campos científicos españoles por actividad.

\subsection{Poco en Sociales y Psique}

Por el contrario, Ciencias sociales y Psicología/Psiquiatría, son los campos cuya producción a nivel mundial es más débil: se alcanzan valores muy por debajo de la media: algo más de un $70 \%$ en Ciencias sociales y menos de la mitad en Psicología. A diferencia con lo ocurrido con los campos que descuellan, aquí sí que se han producido significativas diferencias a lo largo del período. Así, si en el quinquenio (1996-2000) Economía, Ingeniería e Informática figuraban claramente en el furgón de cola, con unas tasas del $50 \%$ por debajo de la media, en el quinquenio 2003-2007 se han situado prácticamente en la media del país. Economía, es el caso más espectacular, por cuanto ha quedado ya bastante por encima. Ha pasado de ser el quinto campo científico por la cola al octavo por la cabeza.

La medida del impacto (número de citas recibidas) de cada campo científico medido en términos de porcentaje relativo respecto al mundo es un indicador muy adecuado para determinar no sólo la visibilidad internacional de cada uno de los campos sino para identificar cuáles de ellos han alcanzado un nivel de excelencia y calidad elevado.

En definitiva un mayor impacto promedio de los artículos de una especialidad será un indicativo cierto de una mayor influencia de nuestro país como potencia científica en dicha rama.
Pues bien, en la tabla 2, que es donde se ordenan los campos científicos por el porcentaje de impacto relativo que poseen respecto al mundo, nos encontramos con un panorama ligeramente diferente al descrito anteriormente. $\mathrm{Y}$ decimos diferente porque podemos constatar cómo el impacto de la producción científica española en la WoS está por debajo de la media mundial: un $-12 \%$ de promedio. No obstante, la progresión también ha sido notable a lo largo del período analizado. Si en 1996-2000 la ciencia española tenía un -17,7\% de impacto respecto al mundo, en 2003-2007 la cifra era ya tan sólo del $-6,3 \%$. Hay que interpretar este hecho muy positivamente. Aparte de este fenómeno general se detectan otros hechos interesantes:

\section{"En 5 años la ciencia española ha aumentado un $11,4 \%$ su impacto respecto al mundo, aunque sigue estando un $12 \%$ por debajo de la media"}

\subsection{Buenos en Física}

Sólo en cuatro áreas de conocimiento hemos figurado siempre por encima del impacto medio del mundo. A saber: Física, Agricultura, Química e Ingeniería. En el caso de la Física se alcanza valores notables $(+26 \%)$; es, además, una especialidad donde el porcentaje de ha multiplicado por dos en los últimos 8 años. Como hemos señalado anteriormente se constatan importantes cambios a lo largo del período ya que si en 1996-2000 sólo 5 campos estaban por encima del impacto medio mundial (Física, Agricultura, Ingeniería, Ciencias del espacio y Química) en 2003-2007 el número había crecido a 8 , incorporándose a este selecto club Medicina clínica,
Ciencia de los materiales, y Ciencia de las plantas y los animales.

\subsection{Sólo bajan Espacio y Socia- les}

Síntoma de la mejoría general es que prácticamente todos los campos científicos han mejorado su impacto a lo largo del período. Sólo dos áreas han empeorado ligeramente: Ciencias del espacio ($2 \%$ ) y Ciencias Sociales (-1\%). En Economía ni se ha avanzado ni se ha retrocedido. En las disciplinas donde más progresos se han producido han sido en: Medicina clínica $(+32 \%)$, Psicología/Psiquiatría $(+27 \%)$, Ecología $(+21 \%)$, Biología \& Bioquímica $(+18 \%)$.

\subsection{Impacto bajo en 5 campos}

En cinco campos estamos en porcentajes de impacto inferiores al 20\% del mundo. Son las áreas donde nuestra investigación alcanza una menor visibilidad: Economía (-31), Psicología/Psiquiatría (-25\%), Informática $(-25 \%)$, Biología molecular $(-21 \%)$ e Inmunología (-20\%). Si bien conviene destacar como Psicología ha mejorado notablemente su posición, igual que lo están haciendo Inmunología y Biología molecular. No es el caso, en cambio, de Economía e Informática. Es llamativo y seguramente ahí encontraremos la explicación a esta situación, cómo eran éstas dos de las disciplinas que más habían crecido. Ese crecimiento en la producción no ha venido acompañado de una mejora en la visibilidad.

De la información contenida en ambas tablas, parece desprenderse cuáles son las fortalezas de la investigación española desde el punto de vista de su peso productivo y de su impacto. Si aceptamos la convención, razonable por otra parte, de que serían aquellas que combinan un peso productivo igual o mayor que la media de España y unos promedios de citación por encima de la media mundial, sólo cuatro áreas científicas cumplen estas condicio- 


\begin{tabular}{|c|c|c|c|c|c|c|c|c|}
\hline Campo científico & $\begin{array}{c}1996- \\
2000 \\
\%\end{array}$ & $\begin{array}{c}1997- \\
2001 \\
\%\end{array}$ & $\begin{array}{c}1998- \\
2002 \\
\%\end{array}$ & $\begin{array}{c}1999- \\
2003 \\
\%\end{array}$ & $\begin{array}{c}2000- \\
2004 \\
\%\end{array}$ & $\begin{array}{c}2001- \\
2005 \\
\%\end{array}$ & $\begin{array}{c}2002- \\
2006 \\
\%\end{array}$ & $\begin{array}{c}2003- \\
2007 \\
\%\end{array}$ \\
\hline Physics & 11 & 12 & 17 & 19 & 25 & 20 & 26 & 26 \\
\hline Agricultural sciences & 10 & 8 & 8 & 7 & 9 & 6 & 10 & 11 \\
\hline Clinical medicine & -21 & -15 & -10 & -5 & 0 & 4 & 6 & 11 \\
\hline Engineering & 3 & 5 & 2 & 5 & 6 & 4 & 8 & 10 \\
\hline Space science & 9 & -7 & -6 & -5 & -4 & -1 & 4 & 7 \\
\hline Materials science & -2 & -2 & -4 & 1 & 3 & 3 & 5 & 6 \\
\hline Chemistry & 1 & 1 & 1 & -1 & 3 & 2 & 4 & 6 \\
\hline Plant $\&$ animal sciences & -12 & -9 & -6 & -9 & -8 & -4 & 1 & 0 \\
\hline Ecology/Environmental & -23 & -17 & -14 & -15 & -9 & -8 & -6 & -2 \\
\hline Mathematics & -16 & -13 & -13 & -6 & -3 & -3 & -7 & -3 \\
\hline Geosciences & -25 & -16 & -14 & -17 & -17 & -12 & -10 & -13 \\
\hline Neuroscience & -24 & -22 & -20 & -18 & -15 & -16 & -13 & -11 \\
\hline Social sciences & -12 & -5 & -18 & -12 & -18 & -6 & -5 & -13 \\
\hline Pharmacology & -28 & -28 & -24 & -21 & -21 & -18 & -15 & -13 \\
\hline Microbiology & -29 & -23 & -22 & -20 & -16 & -19 & -19 & -16 \\
\hline Biology \& biochemistry & -35 & -33 & -29 & -29 & -27 & -25 & -22 & -17 \\
\hline Immunology & -35 & -30 & -28 & -27 & -26 & -21 & -21 & -20 \\
\hline Molecular biology & -35 & -33 & -25 & -10 & -17 & -16 & -17 & -21 \\
\hline Computer science & -26 & -28 & -31 & -28 & -28 & -19 & -19 & -25 \\
\hline Psychology/Psychiatry & -52 & -47 & -44 & -40 & -36 & -31 & -29 & -25 \\
\hline Economics \& Business & -31 & -37 & -37 & -33 & -39 & -35 & -37 & -31 \\
\hline Promedio España & $-17,7$ & $-16,1$ & $-15,1$ & $-12,6$ & $-11,3$ & $-9,3$ & $-7,4$ & $-6,3$ \\
\hline
\end{tabular}

Tabla 2. Impacto relativo de los artículos publicados por España en la WoS distribuidos según campos científicos respecto (1996-2007).

Fuente de datos: ScienceWatch.com

http://in-cites.com/research/2001/april_30_2001-2.html http://in-cites.com/research/2002/may_20_2002-1.html http://in-cites.com/research/2003/june 23 2003-2.html http://in-cites.com/research/2004/july 12 2004-2.html http://in-cites.com/research/2005/july_25_2005-1.html http://in-cites.com/research/2007/september_3_2007-2.html

nes: las Ciencias del Espacio, la Agricultura, la Química y la Física, véanse las figuras 1 y 2.

Las razones que explican este éxito exceden con mucho los objetivos de este comentario. Baste con apuntar un factor común: la sólida tradición científica de estas especialidades en nuestras instituciones académicas, tanto en la universidad como en el CSIC, al que se unen hechos más puntuales como el secular interés por la investigación en la agricultura en este país o la famosa "calidad" de los cielos españoles para la observación astronómica, que ha significado la instalación de numerosos observatorios internacionales en nuestro suelo. En un escalón inmediatamente inferior habría que situar a las Ingenierías y a la Medicina clínica: en la primera porque se ha producido un espectacular incremento productivo manteniendo unos saldos de citación positivos; y en la segunda porque aunque el incremento productivo es modesto su citación ha aumentado en más de $30 \%$, la que más de todas las especialidades.

En el debe hay que anotar el escaso peso productivo de las Ciencias sociales, aunque nos atrevemos a profetizar que será enjugado en buena medida en los próximos años, merced a la rápida incorporación de revistas españolas de este ámbito a la base de datos. Y el relativo fraca- so de la Economía y de la Computación, cuyos fulgurantes ascensos productivos se han revelado poco eficaces para mejorar la visibilidad de los trabajos.

\section{Precauciones}

Terminamos recordando lo que es ya un lugar común en estos análisis, esto es, las limitaciones.

En primer lugar, los bibliomé-

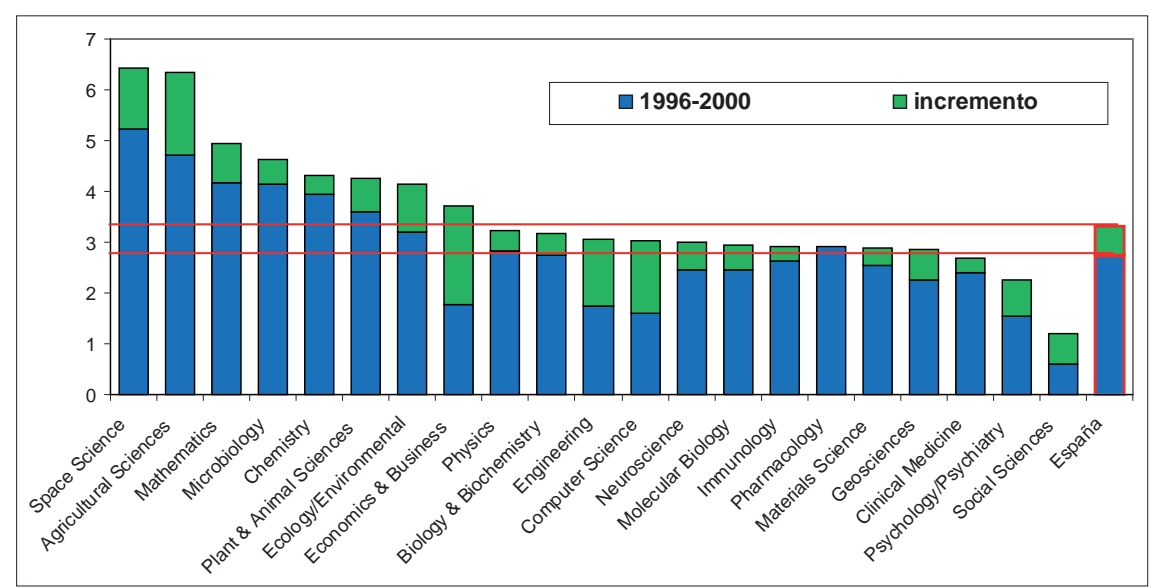

Figura 1. Ordenación de las áreas científicas de acuerdo con su peso total en el último quinquenio. En color verde se marca el incremento entre el primer y último períodos 


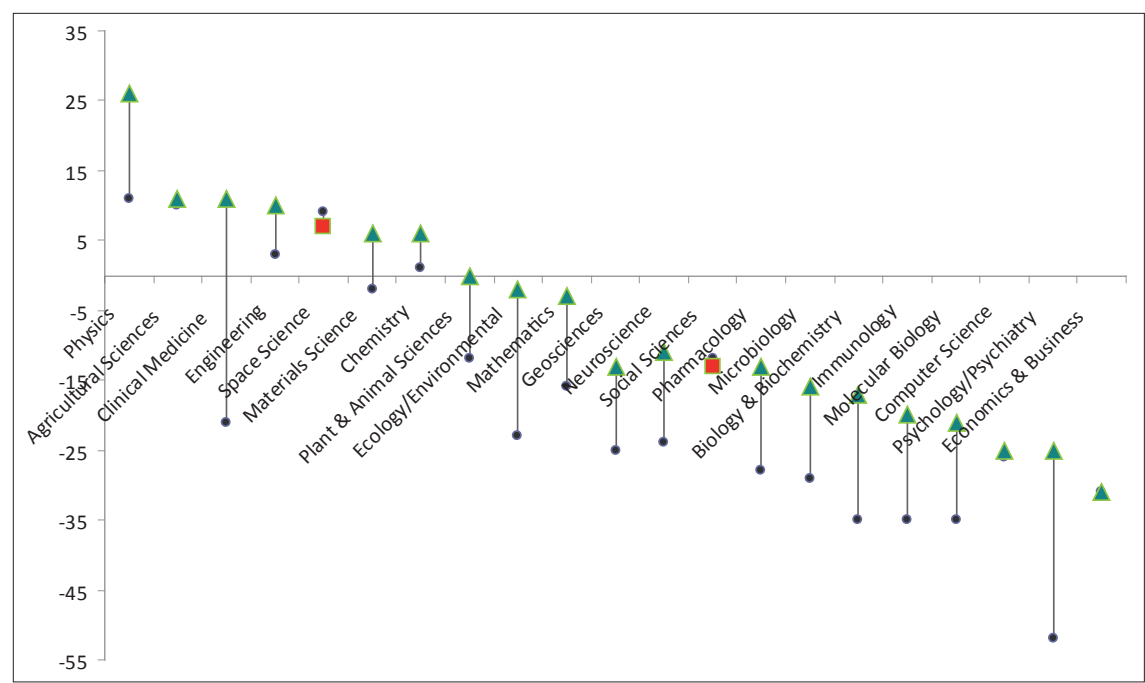

Figura 2. Ordenación de las áreas científicas de acuerdo con su porcentaje de citación. El recorrido de la línea señala el incremento porcentual de la citación. El 0 es la media mundial

tricos son indicadores parciales $\mathrm{y}$ deben combinarse con otros datos; el análisis realizado en este trabajo se refiere exclusivamente a los resultados científicos y no considera los recursos (humanos o económicos) puestos en marcha para alcanzar esta situación y que seguramente explicarán al menos en parte los resultados descritos. La actividad científica es una actividad económica y social más: por tanto, hay que relacionarla con los principales indicadores socioeconómicos. Tradicionalmente se viene haciendo con tres indicadores: población total, gasto total en I+D+I y, sobre todo, personal dedicado a la investigación medido en equivalentes a jornada completa. Cuando se relativiza la producción científica con estos indicadores se obtiene una visión más objetiva, y seguramente más útil de la productividad (qué resultados con qué recursos) y competitividad o eficiencia (obtener más con menos) de un país. Queda para otro trabajo realizar estas ponderaciones.

En segundo lugar en Bibliometría nunca se debe olvidar que los resultados son directamente proporcionales a la cobertura y calidad de la fuente de donde se extraen los datos (Moed et al. 1995). En el caso del WoS, aun tratándose de una selección de revistas que representa lo mejor de la ciencia mundial, no debemos perder de vista los sesgos de que adolece: hacia la ciencia básica y hacia el mundo anglosajón, además del ya mencionado contrario a las Ciencias sociales y Humanidades. Pero aun con estas limitaciones, el WoS sigue siendo el marco de referencia para el seguimiento de la actividad científica internacional y en ella los ejes centrales de la ciencia, aquellos sobre los que gira el progreso científico y tecnológico, están perfectamente representados.

\section{Bibliografía}

Ruiz-Pérez, Rafael; Jiménez-Contreras, Evaristo; Delgado-López-Cózar, Emilio. "Complementos bibliométricos de Thomson Scientific en la Web: buenos, bonitos y gratuitos". El profesional de la información, 2008, v. 17, n. 5, pp. 553-557.

Jiménez-Contreras, Evaristo; Delgado-LópezCózar, Emilio; Ruiz-Pérez, Rafael. "Producción española en biblioteconomía y documentación con visibilidad internacional a través del Web of Science (1995-2004)". El profesional de la información, 2006, v. 15, n. 5, pp. 373-383.

Jiménez-Contreras, Evaristo; Moya-Anegón, Félix; Delgado-López-Cózar, Emilio. "The evolution of research activity in Spain. The impact of the National Commission for the Evaluation of Research Activity (Cneai)". Research policy, 2003, v. 32, n. 1, pp. 123-142.

Pérez-Andrés, Cristina; Delgado-López-Cózar, Emilio; Jiménez-Contreras, Evaristo. "La Revista española, de salud pública ingresa en el Social science citation index de Thomson Scientific". Revista española de salud pública, 2006, v. 80, n. 4, pp. 293-302.

Delgado-López-Cózar, Emilio; Jiménez-Contreras, Evaristo; Ruiz-Pérez, Rafael. "España y los 25 grandes de la ciencia mundial en cifras (1992-2008)". El profesional de la información, 2009, v. 18 , n. 1 , pp. 81-86.

Moed, Henk F.; Van Leeuwen, Thed N. Improving the accuracy of the Institute for Scientific Information journal impact factors". Journal of the American Society for Information Science, 1995, v. 46, n. 6, pp. 461-467.

\section{Emilio Delgado-López-Cózar*, Evaristo Jiménez-Contreras y Ra- fael Ruiz-Pérez.}

Evaluación de la Ciencia y de la Comunicación Científica (EC3), Departamento de Biblioteconomía y Documentación, Universidad de Granada, Campus Cartuja.

18071 Granada

*Responsable de la correspondencia

edelgado@ugr.es

evaristo@ugr.es

rruiz@ugr.es

\section{Suscripción EPI sólo online}

Pensando sobre todo en los posibles suscriptores latinoamericanos, ya no es obligatorio pagar la suscripción impresa de EPI para acceder a la online.

EPI se ofrece a instituciones en suscripción "sólo online"

a un precio considerablemente más reducido (90 euros/año), puesto que en esta modalidad no hay que cubrir los gastos de imprenta ni de correo postal. 


\section{Rapidez, precisión, flexibilidad}

En el siglo XXI, más que nunca, la información es poder. Ayudar a empresas e instituciones a gestionar y rentabilizar su caudal informativo, tanto el que poseen como el que genera diariamente su actividad, es el objetivo y la razón de ser de Baratz.

\section{www.baratz.es}

\section{0 informa@baratz.es}

